H. SUMI

KODAI MATH. J.

21 (1998), 10-28

\title{
ON HAUSDORFF DIMENSION OF JULIA SETS OF HYPERBOLIC RATIONAL SEMIGROUPS
}

\author{
HIROKI SUMI*
}

\begin{abstract}
We will show that if a semigroup of rational functions on the Riemann sphere is finitely generated, then the hyperbolicity and the expandingness are equivalent. Also we consider finitely generated rational semigroups satisfying the strong open set condition. We show that if a semigroup satisfies the strong open set condition, we can construct a $\delta$-conformal measure on the Julia set. Also the Julia set has no interior points, and furthurmore, if the semigroup is hyperbolic, the Hausdorff dimension of the Julia set is strictly lower than 2 . The value $\delta$ of the dimension coincides with the unique value that allows us to construct a $\delta$-conformal measure and the $\delta$-Hausdorff measure of the Julia set is a finite value strictly bigger than zero.

With the method similar to that of the construction of the Patterson-Sullivan measures we get $\delta$-subconformal measures in more general cases and we will show that if a finitely generated rational semigroup is expanding, then the Hausdorff dimension of the Julia set is less than the exponent $\delta$.
\end{abstract}

\section{Introduction}

For a Riemann surface $S$, let $\operatorname{End}(S)$ denote the set of all holomorphic endomorphisms of $S$. It is a semigroup with the semigroup operation being composition of functions. A rational semigroup is a subsemigroup of $\operatorname{End}(\bar{C})$ without any constant elements. Similarly, an entire semigroup is a subsemigroup of $\operatorname{End}(\boldsymbol{C})$ without any constant elements. A rational semigroup $\boldsymbol{G}$ is called a polynomial semigroup if each $g \in G$ is a polynomial. When a rational or entire semigroup $G$ is generated by $\left\{f_{1}, f_{2}, \ldots, f_{n}, \ldots\right\}$, we denote this situation by

$$
G=\left\langle f_{1}, f_{2}, \ldots, f_{n}, \ldots\right\rangle \text {. }
$$

A rational or entire semigroup generated by a single function $g$ is denoted by $\langle g\rangle$. We denote the $n$-th iterate of $f$ by $f^{n}$.

The studies of dynamics of rational semigroups were introduced by $\mathbf{W}$. Zhou and F. Ren $[\mathrm{ZR}], \mathrm{Z}$. Gong and F. Ren [GR] and Hinkkanen and Martin

\footnotetext{
Key words: hyperbolic rational semigroup, strong open set condition, $\delta$-(sub)conformal measure * Partially supported by JSPS Research Fellowships for Young Scientists.

Received April 30, 1997; revised January 26, 1998.
} 
[HM1]. Some properties of dynamics of rational semigroups were studied in [HM1], [HM2], [S1] and [S2]. In [S3], dynamics of hyperbolic rational semigroups are investigated and it is shown that all the limit functions of finitely generated rational semigroups on the Fatou sets are constant functions that take their values in the post critical sets. Also with respect to pertubations of generators of any finitely generated hyperbolic rational semigroup, the hyperbolicity is kept and the Julia set moves continuously.

In this paper, we will show that if a finitely generated rational semigroup contains an element of degree at least two and each Möbius transformation in it is neither the identity nor an elliptic element, then the hyperbolicity and expandingness are equivalent. If the sets of backward images of the Julia set by generators are almost disjoint, then the Julia set has no interior points. We construct a generalized $\delta$-conformal measure on the Julia set of any rational semigroup which satisfies the strong open set condition. We show that if the semigroup is hyperbolic, then the Hausdorff dimension of the Julia set coincides with the unique value $\delta$ that allows us to construct a $\delta$-conformal measure and it is strictly less than 2. Also the $\delta$-Hausdorff measure of the Julia set is a finite value strictly bigger than zero. Considering the convergent series of the norm of the derivative at the backward images, with the method similar to that of the construction of the Patterson-Sullivan measures on the limit sets of Kleinian groups we get a $\delta$-subconformal measure in more general case and we will show that if a finitely generated rational semigroup is expanding, then the Hausdorff dimension of the Julia set is less than the exponent $\delta$.

Generalized Brolin-Lyubich's invariant measures on the Julia set of any rational semigroup which is hyperbolic or satisfying the strong open set condition are constructed in [S4] and a lower estimate of the Hausdorff dimension of the rational semigroups is given.

The author will discuss about the existence and uniqueness of the conformal measures and self-similar measures of rational semigroups in more general cases ([S5]). In that paper we use the thermodynamic formalism and give an upper bound of the Hausdorff dimension of the Julia sets of finitely generated hyperbolic rational semigroups.

In [S8], we will investigate the dynamics of sub-hyperbolic and semi-hyperbolic rational semigroups. We will show some non-wandering domain theorems.

The summary of [S5] and [S8] is in [S7].

Acknowledgement. The author would like to express his gratitude to Prof. S. Ushiki, Prof. M. Taniguchi, Prof. J. Kigami, Prof. T. Sugawa and Prof. M. Kisaka for many valuable discussions and advices. The author especially would like to express his gratitude to the referee for many valuable and helpful advices.

DeFINITION 1.1. Let $G$ be a rational semigroup.

$$
\begin{gathered}
F(G) \stackrel{\text { def }}{=}\{z \in \bar{C} \mid G \text { is normal in a neighborhood of } z\}, \\
J(G) \stackrel{\text { def }}{=} \bar{C} \backslash F(G) .
\end{gathered}
$$


$F(G)$ is called the Fatou set for $G$ and $J(G)$ is called the Julia set for $G$. Similarly, the Fatou set and the Julia set for any entire semigroup are defined.

DEFINITION 1.2. Let $G$ be a rational semigroup and $z$ be a point of $\bar{C}$. The backward orbit $\mathrm{O}^{-}(z)$ of $z$ and the set of exceptional points $E(G)$ are defined by:

$$
\begin{gathered}
O^{-}(z) \stackrel{\text { def }}{=}\{w \in \bar{C} \mid \text { there is some } g \in G \text { such that } g(w)=z\}, \\
E(G) \stackrel{\text { def }}{=}\left\{z \in \bar{C} \mid \sharp O^{-}(z) \leq 2\right\} .
\end{gathered}
$$

DefintTion 1.3. A subsemigroup $H$ of a semigroup $G$ is said to be of finite index if there is a finite collection of elements $\left\{g_{1}, g_{2}, \ldots, g_{n}\right\}$ of $G$ such that $G=\bigcup_{l=1}^{n} g_{i} H$. Similarly we say that a subsemigroup $H$ of $G$ has cofinite index if there is a finite collection of elements $\left\{g_{1}, g_{2}, \ldots, g_{n}\right\}$ of $G$ such that for every $g \in G$ there is $j \in\{1,2, \ldots, n\}$ such that $g_{j} g \in H$.

Next lemma was shown in [S3].

LEMMA 1.4. Let $G$ be a rational semigroup.

1. For any $f \in G$,

$$
\begin{gathered}
f(F(G)) \subset F(G), \quad f^{-1}(J(G)) \subset J(G), \\
F(G) \subset F(\langle f\rangle), \quad J(\langle f\rangle) \subset J(G) .
\end{gathered}
$$

2. If $G=\left\langle f_{1}, \ldots, f_{n}\right\rangle$, then

$$
F(G)=\bigcap_{i=1}^{n} f_{i}^{-1}(F(G)), \quad J(G)=\bigcup_{l=1}^{n} f_{l}^{-1}(J(G)) .
$$

If a set $K$ satisfies that $K=\bigcup_{i=1}^{n} f_{i}^{-1}(K)$, we say that $K$ has backward selfsimilarity.

Next lemma was shown in [HM1].

LEMMA 1.5. Let $G$ be a rational semigroup.

1. If a subsemigroup $H$ of $G$ is of finite or cofinite index, then

$$
J(H)=J(G) .
$$

In particular, when $G$ is a rational semigroup generated by finite elements $\left\{f_{1}, f_{2}, \ldots, f_{n}\right\}$ and $m$ is an integer, if we set

$$
\begin{gathered}
H_{m}=\left\{g=f_{j_{1}} \cdots f_{j_{k}} \in G \mid m \text { devides } k\right\} \\
I_{m}=\{g \in G \mid g \text { is a product of some elements of word length } m\}
\end{gathered}
$$

then

$$
J(G)=J\left(H_{m}\right)=J\left(I_{m}\right) .
$$

Here we say an element $f \in G$ is word length $m$ if $m$ is the minimum integer such that

$$
f=f_{j_{1}} \cdots f_{j_{m}} \text {. }
$$


2. If $J(G)$ contains at least three points, then $J(G)$ is a perfect set.

3. If there is some $g \in G$ such that $\operatorname{deg}(g) \geq 2$ or there is some $g \in G$ such that $\operatorname{deg}(g)=1$ and the order of $g$ is infinite, then

$$
\sharp E(G) \leq 2 \text {. }
$$

4. If a point $z$ is not in $E(G)$, then for every $x \in J(G), x$ belongs to $\overline{O^{-}(z)}$. In particular if a point $z$ belongs to $J(G) \backslash E(G)$, then

$$
\overline{O^{-}(z)}=J(G) \text {. }
$$

5. If there is some $g \in G$ such that $\operatorname{deg}(g) \geq 2$ or there is some $g \in G$ such that $\operatorname{deg}(g)=1$ and the order of $g$ is infinite and $J(G)$ contains at least three points, then $J(G)$ is the smallest closed backward invariant set containing at least three points. Here we say that a set $A$ is backward invariant under $G$ if for each $g \in G$, $g^{-1}(A) \subset A$.

6. If $J(G)$ contains at least three points, then

$$
J(G)=\overline{\{z \in \bar{C} \mid z \text { is a repelling fixed point of some } g \in G\}} \text {. }
$$

Remark 1. A similar result of 6 . for entire semigroup can also be stated.

\section{Hyperbolicity and strong open set condition}

Definition 2.1. Let $G$ be a rational semigroup. We set

$$
P(G)=\bigcup_{g \in G}\{\text { critical values of } g\}
$$

and we say that $G$ is hyperbolic if $P(G) \subset F(G)$. We call $P(G)$ the post critical set of $G$.

DEFINITION 2.2. Let $G=\left\langle f_{1}, f_{2}, \ldots, f_{n}\right\rangle$ be a finitely generated rational semigroup. We say that $G$ satisfies the strong open set condition if there is an open neighborhood $O$ of $J(G)$ such that each set $f_{J}^{-1}(O)$ is included in $O$ and is mutually disjoint.

The Julia set of a rational semigroup may have non-empty interior points in general. For example, the Julia set of $\left\langle z^{2}, 2 z\right\rangle$ is the closure of the unit disc. In [HM2], it was shown that if $G$ is a finitely generated rational semigroup, then each super attracting fixed point of any element of $g \in G$ does not belong to the boundary of the Julia set. So we can construct many examples such that the Julia set has non-empty interior points. Here we show a sufficient condition that the Julia set has no interior points.

THEOREM 2.3. Let $G=\left\langle f_{1}, f_{2}, \ldots, f_{n}\right\rangle$ be a finitely generated rational semigroup. We assume that the set $\bigcup_{(i, j): t \neq J} f_{l}^{-1}(J(G)) \cap f_{J}^{-1}(J(G))$ does not contain any continuum. Then the Julia set $J(G)$ has no interior points. 
Proof. Assume $J(G)$ has non-empty interior points and let $U$ be a component of $\operatorname{int}(J(G))$. Let $x$ be a point of $U$. From Lemma 1.4.2, there exists a positive integer $i_{1}$ with $i_{1} \leq n$ such that $x \in f_{i_{1}}^{-1}(J(G))$. From Lemma 1.5.4, we have $U \cap f_{l_{1}}^{-1}$ (int $\left.J(G)\right) \neq \emptyset$. Let $V$ be the connected component of $U \cap f_{l_{1}}^{-1}(\operatorname{int} J(G))$.

We will show that $V$ is dense in $U$. To show that, we can assume that $V \neq U$. Then $\partial V \cap U \neq \emptyset$. If $\partial V \cap U$ contains a continuum $K$, then from the assumption of our theorem, there exists a point $z \in K$ such that for each $j$ with $j \neq i_{1}, z \notin f_{j}^{-1}(J(G))$. We denote the open disk centered at $z$ of radius $\varepsilon$ by $D(z, \varepsilon)$. Hence there is a small positive number $\varepsilon$ such that $D(z, \varepsilon)$ is included in $U$ and disjoint from $\bigcup_{J \neq l_{1}} f_{J}^{-1}(J(G))$. From Lemma 1.4.2, $D(z, \varepsilon) \subset$ $f_{l_{1}}^{-1}$ (int $\left.J(G)\right)$ and this is a contradiction because $V$ is a connected component of $U \cap f_{t_{1}}^{-1}($ int $J(G))$. Therefore $\partial V \cap U$ does not contain any continuum and $V$ is dense in $U$.

It follows that $f_{i_{1}}(U)$ is included in a component $U_{1}$ of $\operatorname{int}(J(G))$. In this way, we can take a sequence $\left(i_{k}\right)_{k}$ such that for each $k$ the number $i_{k}$ is in $\{1, \ldots, n\}$ and

$$
f_{i_{k}} \circ \cdots \circ f_{i_{1}}(U) \subset U_{k}
$$

where $U_{k}$ is a component of $\operatorname{int}(J(G))$. Now let $\left(g_{j}\right)$ be a sequence of elements of $G$. If the sequence contains infinite elements of $\left(f_{i_{k}} \circ \cdots \circ f_{i_{1}}\right)$, then $\left(g_{j}\right)$ is a normal family on $U$. Unless $\left(g_{j}\right)$ contains any element of the form $f_{i_{k}} \circ \cdots \circ f_{i_{1}}$, then for each $l$ the set $g_{l}(U)$ is included in $F(G)$ because of the assumption of our theorem and so $\left(g_{j}\right)$ is a normal family on $U$. It follows that $U$ is included in $F(G)$ and this is a contradiction.

Remark 2. If $\bigcup_{(i, j): l \neq j} f_{l}^{-1}(J(G)) \cap f_{j}^{-1}(J(G))$ contains a continuum, then the Julia set may have non-empty interior points. For example, let $p_{1}=0$, $p_{2}=1, p_{3}=1+i$ and $p_{4}=i$. For each $j=1, \ldots, 4$, we set $f_{j}(z)=2\left(z-p_{j}\right)+$ $p_{j}$. Then $J\left(\left\langle f_{1}, \ldots, f_{4}\right\rangle\right)$ is equal to the closed rectangle $p_{1} p_{2} p_{3} p_{4}$.

Definition 2.4. Let $G$ be a polynomial semigroup. We denote by $K(G)$ the closure of the set $K_{1}(G)$ consisting of the points, for each $z$ of which, there is a sequence $\left(g_{m}\right)_{m}$ consisting of mutually distinct elements of $G$ such that the sequence $\left(g_{m}(z)\right)_{m}$ is bounded. $K(G)$ is called the filled-in Julia set of $G$.

Remark 3. For each $g \in G$ the inverse image $g^{-1}(K(G))$ is included in $K(G)$ and $J(G) \subset K(G)$. If $G=\left\langle f_{1}, f_{2}, \ldots, f_{n}\right\rangle$ is a finitely generated polynomial semigroup, then

$$
K(G)=\bigcup_{J=1}^{n} f_{J}^{-1}(K(G)) .
$$

THEOREM 2.5. Let $G=\left\langle f_{1}, f_{2}, \ldots, f_{n}\right\rangle$ be a finitely generated polynomial semigroup. Assume that the set $\bigcup_{(i, j): l \neq J} f_{i}^{-1}(K(G)) \cap f_{J}^{-1}(K(G))$ does not 
contain any continuum. Then

$$
\partial(K(G))=J(G)
$$

Proof. Let $z$ be a point of $\partial(K(G))$ and let $U$ be an open neighborhood of $z$. For each $x \in K_{1}(G) \cap U$ there is a sequence $\left(g_{m}\right)_{m}$ of elements of $G$ such that $\left(g_{m}(x)\right)_{m}$ is bounded. But for each $y \in U \backslash K(G)$ the sequence $\left(g_{m}(y)\right)_{m}$ tends to infinity so $G$ is not normal in $U$ and $z \in J(G)$. So $\partial(K(G)) \subset J(G)$. Next let $U$ be a component of $\operatorname{int}(K(G))$. From the fact $K(G)=\bigcup_{J=1}^{n} f_{J}^{-1}(K(G))$ and our assumption we can show that $G$ is normal in $U$ in the same way as the proof of Theorem 2.3.

Now we consider the expandingness of hyperbolic rational semigroups, which gives us an information about the analytic property of them.

THEOREM 2.6. Let $G=\left\langle f_{1}, f_{2}, \ldots, f_{n}\right\rangle$ be a finitely generated hyperbolic rational semigroup. Assume that $G$ contains an element with the degree at least two and each Möbius transformation in $G$ is neither the identity nor an elliptic element. Let $K$ be a compact subset of $\bar{C} \backslash P(G)$. Then there are a positive number $c, a$ number $\lambda>1$ and a Riemannian metric $\rho$ on an open subset $V$ of $\bar{C} \backslash P(G)$ which contains $K \cup J(G)$ and is backward invariant under $G$ such that for each $k$

$$
\inf \left\{\left\|\left(f_{i_{k}} \circ \cdots \circ f_{i_{1}}\right)^{\prime}(z)\right\|_{\rho} \mid z \in\left(f_{i_{k}} \circ \cdots \circ f_{i_{1}}\right)^{-1}(K),\left(i_{k}, \ldots, i_{1}\right) \in\{1, \ldots, n\}^{k}\right\}
$$

$\geq c \lambda^{k}$, here we denote by $\|\cdot\|_{\rho}$ the norm of the derivative measured from the metric $\rho$ to it.

Proof. We will show the statement in the way similar to that of the proof of Theorem 3.13 in [M]. We denote by $B$ the union of all components of $F(G)$ each of which has a non-empty intersection with $P(G)$. Let $B_{1}, \ldots, B_{s}$ be all the components of $B$. For each $j=1, \ldots, s$ we take the hyperbolic metric in $B_{j}$. Let $L_{j}$ be the $\varepsilon$-neighborhood of $P(G) \cap B_{j}$ in $B_{j}$ with respect to the distance in $B_{j}$ induced by the hyperbolic metric, where $\varepsilon$ is a positive number which is sufficiently small. We set $L=\bigcup_{J=1}^{s} L_{J}$ and $V=\bar{C} \backslash L$. Then $V$ contains $K \cup$ $J(G)$ and for each element $g$ of $G$ the inverse image $g^{-1}(V)$ is included in $V$.

We see that for large positive integer $m$, every element of $G$ which is a product of $m$ generators of $G$ is a contraction map from $B$ to $B$ and the contraction rate is bounded by a constant strictly less than one in each fixed compact subset of $B$. For, assume that $g \in G$ is of the form $f_{i_{s}} \circ \cdots \circ f_{i_{1}}$. For each $j$ there are positive integers $u, v$ with $u<v$ which are smaller than $s$ such that $f_{i_{v}} \circ \cdots \circ f_{i_{1}}\left(B_{j}\right)=f_{i_{u}} \circ \cdots \circ f_{i_{1}}\left(B_{j}\right)$. Hence $f_{i_{v}} \circ \cdots \circ f_{i_{u+1}}$ maps the component $U$ of $F(G)$ which contains $f_{i_{u}} \circ \cdots \circ f_{i_{1}}\left(B_{j}\right)$ into it. This map is a contraction with respect to the hyperbolic metric on $U$ and the contraction rate is bounded by a constant strictly less than one in each fixed compact subset of $U$, 
because of the assumpsion of our theorem. Thus $g$ is a contraction map from $B_{j}$ to the component of $F(G)$ which contains $g\left(B_{j}\right)$ and the above claim holds.

So there is a positive integer $m_{0}$ such that for each number $m \geq m_{0}$ the closure of $g_{m}^{-1}(V)$ is included in $V$ for any element $g_{m}$ of $G$ in the form $f_{i_{m}} \circ \cdots \circ f_{i_{1}}$. Now let $m$ be any positive integer with $m \geq m_{0}$ and $g_{m}$ any element of $G$ in the form $f_{i_{m}} \circ \cdots \circ f_{i_{1}}$. We set $U=g_{m}^{-1}(V)$. We take the hyperbolic metric in each component of $V$ and denote it by $\rho$. Also we take the hyperbolic metric in each component of $U$ and denote it by $\tau$.

We will show that the inclusion map $i: U \rightarrow V$ satisfies that $\left\|i^{\prime}(z)\right\|<1$ for each $z \in U$ where we denote by $\|\cdot\|$ the norm of the derivative measured from the Riemannian metric $\tau$ on $U$ to the Riemannian metric $\rho$ on $V$. Assume that there is a point $z_{0} \in U$ such that $\left\|i^{\prime}\left(z_{0}\right)\right\|=1$. Let $W_{1}$ be the connected component of $U$ containing $z_{0}$ and $W_{2}$ the connected component of $V$ containing $z_{0}$. For each $i=1,2$ the universal cover of $W_{\imath}$ is $D(0,1)$. Let $\tilde{i}: D(0,1) \rightarrow D(0,1)$ be the lift of $i: W_{1} \rightarrow W_{2}$. Since $\left\|i^{\prime}\left(z_{0}\right)\right\|=1, \tilde{i}(D(0,1))=D(0,1)$ from Schwartz lemma. It follows that $W_{1}=W_{2}$ but this is a contradiction because the closure of $g_{m}^{-1}(V)$ is included in $V$. Hence $\left\|i^{\prime}(z)\right\|<1$ for each $z \in U$.

The map $g_{m}$ is a covering map from $U$ to $V$ and is a local isometry between the Riemannian metric $\tau$ on $U$ and $\rho$ on $V$. Hence $\left\|g_{m}^{\prime}(z)\right\|_{\rho}>1$ for each $z \in U$, where we denote by $\|\cdot\|_{\rho}$ the norm of the derivative measured from the Riemannian metric $\rho$ on $V$ to it.

It is eacy to see that there exists a compact subset $C$ of $V$ which contains $K$ and is backward invariant under $G$, hence the statement of our theorem holds.

COROLLARY 2.7. Under the same assumpsion of Theorem 2.6, if $W$ is a simply connected domain which is a relatively compact subdomain of $\bar{C} \backslash P(G)$ and $\mathscr{A}$ is a family of maps on $W$ such that each element $h$ of $\mathscr{A}$ is a well defined branch of $g^{-1}$ where $g$ is an element of $G$, then each limit function of $\mathscr{A}$ on $W$ is a constant function such that the constant value is in $J(G)$.

Proof. By Theorem 2.6, each limit function of $\mathscr{A}$ on $W$ is constant. And by [S3], for each point $z$ of $F(G)$, the $G$-orbit of $z$ can accumulate only to $P(G)$. Since $\bar{W} \subset \bar{C} \backslash P(G)$, the constant values belong to $J(G)$.

Now we will show the converse of Theorem 2.6.

THEOREM 2.8. Let $G=\left\langle f_{1}, f_{2}, \ldots, f_{n}\right\rangle$ be a finitely generated rational semigroup. If there are a positive number $c$, a number $\lambda>1$ and a Riemannian metric $\rho$ on an open subset $U$ containing $J(G)$ such that for each $k$

$$
\inf \left\{\left\|\left(f_{i_{k}} \circ \cdots \circ f_{i_{1}}\right)^{\prime}(z)\right\|_{\rho} \mid z \in\left(f_{i_{k}} \circ \ldots \circ f_{i_{1}}\right)^{-1}(J(G)),\left(i_{k}, \ldots, i_{1}\right) \in\{1, \ldots, n\}^{k}\right\}
$$

$\geq c \lambda^{k}$, where we denote by $\|\cdot\|_{\rho}$ the norm of the derivative measured from the metric $\rho$ on $V$ to it, then $G$ is hyperbolic and each Möbius transformation in $G$ is loxodromic. 
Remark 4. Because of the compactness of $J(G)$, we can show, with an easy argument, which is familiar to us in the iteration theory of rational functions, that even if we exchange the metric $\rho$ to another Riemannian metric $\rho_{1}$, the inequality of the assumption holds with the same number $\lambda$ and a different constant $c_{1}$.

Proof. Take a positive integer $k$ such that $c \lambda^{k}>1$ and fix it. We take the compact $\varepsilon$-neighborhood $K$ of $J(G)$ in $U$ with respect to the distance $\rho$. If $\varepsilon$ is sufficiently small, then

$$
\inf \left\{\left\|\left(f_{i_{k}} \circ \cdots \circ f_{i_{1}}\right)^{\prime}(z)\right\|_{\rho} \mid z \in\left(f_{i_{k}} \circ \cdots \circ f_{i_{1}}\right)^{-1}(K),\left(i_{k}, \ldots, i_{1}\right) \in\{1, \ldots, n\}^{k}\right\}
$$

$>1$ and for each $g \in G$ which is of the form $f_{i_{k}} \circ \cdots \circ f_{i_{1}}$, the set $g^{-1}(K)$ is included in $K$. Moreover if we take $\varepsilon$ smaller, then in $K$ there is no critical value of any element of $G$ with the word length less than $k$ hence there is no critical value of any element of $G$ in $K$.

Now let $h$ be any Möbius transformation in $G$. We will show that $h$ is loxodromic. Assume that $h$ is parabolic. Then the fixed point $x$ of $h$ satisfies that $\left(h^{n}\right)^{\prime}(x)=1$ for each positive integer $n$ and $x \in J(G)$ but this is a contradiction. Assume that $h$ is elliptic. We can assume that $h(z)=e^{i \theta} z$ for some $\theta \in R$ and $C \cap J(G) \neq \emptyset$. There is a sequence $\left(n_{j}\right)$ of positive integers such that for $y \in C \cap J(G), h^{n_{j}}(y) \rightarrow y$ and $\left(h^{n j}\right)^{\prime}(y) \rightarrow 1$ as $j \rightarrow \infty$ but this is a contradiction.

DeFINITION 2.9. Let $G=\left\langle f_{1}, f_{2}, \ldots, f_{n}\right\rangle$ be a finitely generated rational semigroup. We say that $G$ is expanding if the assumption of Theorem 2.8 holds.

THEOREM 2.10. Let $G=\left\langle f_{1}, f_{2}, \ldots, f_{n}\right\rangle$ be a finitely generated hyperbolic rational semigroup satisfying the strong open set condition. Then $m(J(G))=0$, where we denote by $m$ the Lebesgue measure on $\bar{C}$.

Proof. We can assume that $\infty$ belongs to $F(G)$. From Theorem 2.6, Lemma 1.5.1 and the remark stated after Theorem 2.8, we can also assume that there is a number $\lambda>1$ such that for each $k$

$$
\inf \left\{\left|f_{k}^{\prime}(z)\right| \mid z \in f_{k}^{-1}(J(G))\right\}>\lambda .
$$

From Theorem 2.3, $J(G)$ has no interior points. We fix a small positive number $\varepsilon$. Then there is a number $1>\delta>0$ such that for each $\zeta \in J(G)$

$$
\frac{m(D(\zeta, \varepsilon) \cap F(G))}{m(D(\zeta, \varepsilon))} \geq \delta
$$

where we denote by $D(\zeta, \varepsilon)$ the $\varepsilon$ disc about $\zeta$. We fix any point $z_{0}$ in $J(G)$. From Lemma 1.4.2, for each integer $j$ there is a unique element $g_{j} \in G$ of word length $j$ such that $g_{j}\left(z_{0}\right)$ belongs to $J(G)$. We denote by $D_{j}$ the component of $g_{j}^{-1}\left(D\left(g_{j}\left(z_{0}\right), \varepsilon\right)\right)$ containing $z_{0}$. From the Koebe theorem, there are positive 
numbers $c_{0}, c_{1}, c_{2}$ such that for every $j$

$$
\begin{gathered}
D\left(z_{0}, c_{0}\left|g_{j}^{\prime}\left(z_{0}\right)\right|^{-1}\right) \subset D_{j} \subset D\left(z_{0}, c_{1}\left|g_{j}^{\prime}\left(z_{0}\right)\right|^{-1}\right) \\
\quad \frac{\inf \left\{\left|\left(g_{j}^{-1}\right)^{\prime}(z)\right| \mid z \in D\left(g_{j}\left(z_{0}\right), \varepsilon\right)\right\}}{\sup \left\{\left|\left(g_{j}^{-1}\right)^{\prime}(z)\right| \mid z \in D\left(g_{j}\left(z_{0}\right), \varepsilon\right)\right\}} \geq c_{2},
\end{gathered}
$$

where we take a branch of $g_{j}^{-1}$ so that it maps $D\left(g_{j}\left(z_{0}\right), \varepsilon\right)$ onto $D_{j}$. Now for each $j$,

$$
\begin{aligned}
& g_{j}\left(D_{j} \cap J(G)\right)=D\left(g_{j}\left(z_{0}\right), \varepsilon\right) \cap J(G), \\
& g_{j}\left(D_{j} \cap F(G)\right)=D\left(g_{j}\left(z_{0}\right), \varepsilon\right) \cap F(G),
\end{aligned}
$$

from Lemma 1.4.2 and because of the strong open set condition. So for each $j$

$$
\frac{m\left(D_{j} \cap F(G)\right)}{m\left(D_{j}\right)}=\frac{\int_{D\left(g_{j}\left(z_{0}\right), \varepsilon\right) \cap F(G)}\left|\left(g_{j}^{-1}\right)^{\prime}(z)\right|^{2} d m}{\int_{D\left(g_{j}\left(z_{0}\right), \varepsilon\right)}\left|\left(g_{j}^{-1}\right)^{\prime}(z)\right|^{2} d m} \geq c_{2}^{2} \delta .
$$

So from (1) for each $j$

$$
\frac{m\left(D\left(z_{0}, c_{1}\left|g_{j}^{\prime}\left(z_{0}\right)\right|^{-1}\right) \cap F(G)\right)}{m\left(D\left(z_{0}, c_{0}\left|g_{j}^{\prime}\left(z_{0}\right)\right|^{-1}\right)\right)} \geq c_{2}^{2} \delta
$$

Also we have

$$
\frac{m\left(D\left(z_{0}, c_{1}\left|g_{j}^{\prime}\left(z_{0}\right)\right|^{-1}\right) \cap F(G)\right)}{m\left(D\left(z_{0}, c_{0}\left|g_{j}^{\prime}\left(z_{0}\right)\right|^{-1}\right)\right)}=\frac{m\left(D\left(z_{0}, c_{1}\left|g_{j}^{\prime}\left(z_{0}\right)\right|^{-1}\right) \cap F(G)\right)}{m\left(D\left(z_{0}, c_{1}\left|g_{j}^{\prime}\left(z_{0}\right)\right|^{-1}\right)\right)} \cdot\left(\frac{c_{1}}{c_{0}}\right)^{2} .
$$

Now $\left|g_{j}^{\prime}\left(z_{0}\right)\right| \rightarrow \infty$ as $j$ tends to infinity and so $z_{0}$ is not the Lebesgue point of $J(G)$. So each point $z \in J(G)$ is not the Lebesgue point of $J(G)$ and $m(J(G))$ is equal to zero.

\section{3. $\delta$-conformal measure}

We construct $\delta$-conformal measures on Julia sets of rational semigroups. $\delta$-conformal measures on Julia sets of rational functions were introduced in [Su1]. See also [MTU].

DeFINITION 3.1. Let $G=\left\langle f_{1}, f_{2}, \ldots, f_{n}\right\rangle$ be a finitely generated rational semigroup satisfying the strong open set condition and let $\delta$ be a non-negative number. We say that a probability measure $\mu$ on $\bar{C}$ is $\delta$-conformal if for each $j=1, \ldots, n$ and for each measurable set $A$ included in $f_{j}^{-1}(J(G))$ where $f_{j}$ is injective on $A$,

$$
\mu\left(f_{j}(A)\right)=\int_{A}\left\|f_{j}^{\prime}(z)\right\|^{\delta} d \mu
$$

where $\|\cdot\|$ denotes the norm of the derivative with respect to the spherical 
metric. And we set

$$
\delta(G)=\inf \{\delta \mid \text { there is a } \delta \text {-conformal measure on } J(G)\} \text {. }
$$

THEOREM 3.2. Let $G=\left\langle f_{1}, f_{2}, \ldots, f_{n}\right\rangle$ be a finitely generated rational semigroup satisfying the strong open set condition. We assume that when $n$ is equal to one the degree of $f_{1}$ is at least two. Then there are a number $0<\delta \leq 2$ and $a$ probability measure $\mu$ whose support is equal to $J(G)$ such that $\mu$ is $\delta$-conformal. Also $\delta(G)>0$.

We will show the statement in the same way as [Sul] or [MTU]. We need the following lemma.

LEMMA 3.3. Under the same assumption in Theorem 3.2, let $O$ be an open set in Definition 2.2. Then there exists an open set $U$ whose closure is included in $O \cap F(G)$ such that for each open neighborhood $W$ of $J(G)$ there is a positive integer $m$ satisfying

$$
\bigcup_{g \in G: \text { word length } \geq m} g^{-1}(U) \subset W
$$

Proof. Let $V$ be an open set whose closure is included in $O \cap F(G)$. First we consider the case such that for each $z \in F(G)$, the $G$-orbit of $z$ does not accumulate at any point of $\bar{V}$. If there are a sequence $\left(x_{k}\right)$ converging to a point $y \in F(G)$ and a sequence $\left(g_{k}\right)$ of $G$ such that for each $k, g_{k}\left(x_{k}\right) \in V$ and the word length of $g_{m}$ tends to infinity as $m \rightarrow \infty$, then the sequence $\left(g_{k}(y)\right)$ accumulates in $\bar{V}$ because $\left(g_{k}\right)$ is normal in a neighborhood of $y$. This is a contradiction. Putting $U=V$, we have (3).

Now let $V_{1}, \ldots, V_{m}$ be all connected components of $F(G)$ each of which has a non-empty intersection with $O^{c}$. For each $j$ we take the hyperbolic metric in $V_{J}$. We set

$$
H=\left\{g \in G \mid g\left(V_{1} \cap O\right) \cap\left(V_{1} \cap O\right) \neq \emptyset\right\} .
$$

If $H$ is empty, take $U$ in $V_{1} \cap O$ so small that whose closure is included in $V_{1} \cap O$. For each $z \in F(G)$, the $G$-orbit of $z$ does not accumulate at any point of $\bar{U}$, so by the previous argument, (3) holds. Hence we can assume that $H$ is non-empty. We have for each $j, f_{j}\left(O^{c}\right) \subset O^{c}$. Therefore for each $h \in H$, $h\left(V_{1} \cap O^{c}\right) \subset V_{1} \cap O^{c}$. If $V_{1}$ is included in a Siegel disc or a Hermann ring of an element of $g \in G$, then $g\left(V_{1} \cap O\right)=V_{1} \cap O$. We can assume the word length of $g$ is less than that of any other element of $G$ which has a Siegel disc or a Hermann ring containing $V_{1}$. We represent $g$ as

$$
g=f_{i_{k}} \cdots f_{i_{1}} \text {. }
$$

Take small open set $U$ in $B=g^{-1}\left(V_{1} \cap O\right) \backslash\left(V_{1} \cap O\right)$. Note that because of the backward self-similarity of $J(G)$ and the strong open set condition, for each open set $D$ in $O \cap F(G)$ and each element $h \in G, h^{-1}(D) \subset O \cap F(G)$ and so $B \subset O \cap$ 
$F(G)$. We have for each $z \in B$,

$$
G(z) \cap O=\left\{f_{i_{s}} \cdots f_{i_{1}} g^{t}(z) \mid 0 \leq s \leq k, t \geq 0\right\} \backslash\{z\} .
$$

Hence $G(z) \cap O$ does not accumulate at any point of $\bar{U}$ and it follows that for each $y \in F(G), G(y)$ does not accumulate at any point of $\bar{U}$. Therefore (3) holds.

So we can assume that each $h \in H$ has a (super)attracting basin containing $V_{1}$, here note that $h\left(V_{1} \cap O^{c}\right) \subset V_{1} \cap O^{c}$. Let $K$ be the compact $\varepsilon$-neighborhood of $O^{c}$ in $\bigcup_{J=1}^{m} V_{J}$ with respect to the hyperbolic metric. For each $j=1, \ldots, n$ and $i=1, \ldots, m$, we set

$$
a_{j i}=\sup \left\{\left\|f_{j}^{\prime}(z)\right\| \mid z \in K \cap V_{l}\right\}
$$

where $\|\cdot\|$ is the norm of the derivative measured from the hyperbolic metric on $V_{l}$ to that on some $V_{u}$ which contains $f_{j}\left(V_{l}\right)$. We denote by $d_{H}(\cdot, \cdot)$ the distance on $V_{1}$ induced by the hyperbolic metric. Then for each $h \in H$ and for each $z \in K \cap V_{1} \cap O, d_{H}\left(h(z), V_{1} \cap O^{c}\right) / d_{H}\left(z, V_{1} \cap O^{c}\right)$ is less than

$$
\sup \left\{a_{j i} \mid j=1, \ldots, n, i=1, \ldots, m, a_{j i} \neq 1\right\}<1 \text {. }
$$

Since $h\left(O^{c}\right) \subset O^{c}$ for each $h \in H$, if we take $U$ small enough in $K \cap V_{1} \cap O$, then for each $y \in F(G), G(y)$ does not accumulate at any point of $\bar{U}$ and so (3) holds.

Proof of Theorem 3.2. Let $O$ be the open set in Definition 2.2. Let $U$ be the open set in Lemma 3.3. We can assume that $U$ is a simply connected domain in $O \backslash(P(G) \cup J(G))$. Now we have

$$
\sum_{S} \int_{U}\left\|S^{\prime}(z)\right\|^{2} d m \leq \infty
$$

where $S$ is taken over all holomorphic inverse branches of all elements of $G$ defined on $U,\|\cdot\|$ denotes the norm of the derivative with respect to the spherical metric and $m$ is the Lebesgue measure. For, assume that there are sequences $\left(m_{k}\right)_{k}$ and $\left(l_{k}\right)_{k}$ of integers with $m_{k} \rightarrow \infty$ such that for each $k$ there is an element $g_{m_{k}+l_{k}} \in G$ of word length $m_{k}+l_{k}$ and $\bar{g}_{l_{k}} \in G$ of word length $l_{k}$ so that

$$
g_{m_{k}+l_{k}}^{-1}(U) \cap \bar{g}_{l_{k}}^{-1}(U) \neq \emptyset \text {. }
$$

Then because of the strong open set condition for each $k$ there is an element $h_{m_{k}} \in G$ of word length $m_{k}$ such that

$$
U \cap h_{m_{k}}^{-1}(U) \neq \emptyset
$$

But this is a contradiction by (3) and so (4) holds.

Now for each $x \in U$ we set

$$
I(x)=\bigcup_{m} \bigcup_{g \in G: \text { word length } m} g^{-1}(x)
$$


and

$$
d(y)=\left\|g^{\prime}(y)\right\|^{-1},
$$

for $y \in g^{-1}(x)$. By (4) for almost everywhere $x \in U$

$$
\sum_{y \in I(x)} d(y)^{2}<\infty
$$

We fix a point $x \in U$ such that (5) holds. And we set

$$
\delta=\inf \left\{s \mid \sum_{y \in I(x)} d(y)^{s}<\infty\right\} .
$$

For each $j$ there is a positive number $C_{j}$ such that $\left\|f_{j}^{\prime}(z)\right\| \leq C_{J}$ in a neighborhood of $f_{j}^{-1}(J(G))$ and the set

$$
\bigcup_{g \in G: \text { word length } m} g^{-1}(x)
$$

has $\left(\sum_{j=1}^{n} \operatorname{deg}\left(f_{j}\right)\right)^{m}$ points so $\delta>0$.

Now we consider the case $\sum_{y \in I(x)} d(y)^{\delta}=\infty$. For each number $s>\delta$ we denote by $\mu_{s}$ the probability measure on $\bar{C}$ such that for each $y \in I(x)$

$$
\mu_{s}(\{y\})=\frac{d(y)^{s}}{\sum_{w \in I(x)} d(w)^{s}} .
$$

Let $\mu$ be a weak limit of $\mu_{s}$ when $s \searrow \delta$. Then the support of $\mu$ is included in $J(G)$ because $\sum_{y \in I(x)} d(y)^{\delta}=\infty$. Let $\zeta$ be a point of $f_{J}^{-1}(J(G))$. Also let $V$ be a neighborhood of $\zeta$ in $f_{J}^{-1}(O)$ and assume that $f_{j}$ is injective on $V$. Then $f_{j}$ is a bijection from $I(x) \cap V$ to $I(x) \cap f_{j}(V)$. We set $\lambda=\left\|f_{j}^{\prime}(\zeta)\right\|$. Let $\varepsilon>0$ be a small number. We take $V$ smaller such that for each $z \in V$

$$
\lambda(1-\varepsilon)<\left\|f_{j}^{\prime}(z)\right\|<\lambda(1+\varepsilon) \text {. }
$$

Then

$$
\lambda^{s} \mu_{s}(V)(1-\varepsilon)^{s} \leq \mu_{s}\left(f_{j}(V)\right) \leq \lambda^{s} \mu_{s}(V)(1+\varepsilon)^{s} .
$$

Let $s \searrow \delta$ and we get

$$
\lambda^{\delta} \mu(V)(1-\varepsilon)^{\delta} \leq \mu\left(f_{j}(V)\right) \leq \lambda^{\delta} \mu(V)(1+\varepsilon)^{\delta} .
$$

If $f_{j}^{\prime}(\zeta)=0$, we can show that $\mu\left(f_{j}(\zeta)\right)=0$. It follows that $\mu$ is a $\delta$-conformal measure on $J(G)$.

Next we consider the case $\sum_{y \in I(x)} d(y)^{\delta}<\infty$. We take Patterson's function $h$ i.e. $h$ is a continuous and non-decreasing function from $\boldsymbol{R}^{+}$to $\boldsymbol{R}^{+}$and satisfies that

1. $\sum_{y \in I(x)} h\left(d(y)^{-1}\right) d(y)^{s}$ converges for each $s>\delta$ and does not converge for each $s \leq \delta$. 
2. for each $\varepsilon$ there is a number $r_{0}$ such that $h(r t) \leq t^{\varepsilon} h(r)$ for each $r>r_{0}$ and $t>1$.

For more detail about Patterson's function, see $[\mathrm{P}]$. We set

$$
\mu_{s}=\frac{1}{\sum_{y \in I(x)} h\left(d(y)^{-1}\right) d(y)^{s}} \sum_{y \in I(x)} h\left(d(y)^{-1}\right) d(y)^{s} \delta_{y},
$$

where we denote by $\delta_{y}$ the dirac measure which is concentrated on $\{y\}$. Letting $s \searrow \delta$ we get a $\delta$-conformal measure on $J(G)$ in the same way as the case $\sum_{y \in I(x)} d(y)^{\delta}=\infty$.

We will show that support of $\mu$ is equal to $J(G)$. By the construction, the support of $\mu$ is included in $J(G)$. Now assume that there are a point $\zeta \in J(G)$ and a positive number $a$ such that $\mu(D(\zeta, a))=0$. By Lemma 1.5, there exists an element $g \in G$ such that $g(D(\zeta, a)) \supset J(G)$. Since $\mu$ is a conformal measure, it follows that $\mu(J(G))=0$ and this is a contradiction. Therefore the support of $\mu$ is equal to $J(G)$.

We now consider $\delta(G)$. There is a $\delta(G)$ conformal measure $\mu$ on $J(G)$. Assume that $\delta(G)$ is equal to zero. If there exists a point $x \in f^{-1}(J(G))$ such that $\mu(\{x\})>0$, then $\mu\left(\left\{f_{j}(x)\right\}\right)=\mu(\{x\})$. Since backward orbit of any point of $J(G)$ has infinitely many points and $\mu$ is a probability measure, it is a contradiction. Hence $\mu$ is non-atomic. For each measurable set $A$ included in $J(G)$ we set

$$
\tau(A)=\mu\left(\bigcup_{J=1}^{n} f_{J}^{-1}(A)\right) .
$$

Then $\tau$ is a probability measure on $J(G)$. But if $A$ is a measurable set in $J(G)$ such that for each $j$ all branches of $f_{j}^{-1}$ are well defined on $A$ then

$$
\tau(A)=\left(\sum_{j=1}^{n} \operatorname{deg}\left(f_{j}\right)\right) \mu(A)
$$

and this is a contradiction, since $J(G)$ is a disjoint union of some finitely many points and some sets on each of which for each $j$ all branches of $f_{j}^{-1}$ are well defined. ,

THEOREM 3.4. Let $G=\left\langle f_{1}, f_{2}, \ldots, f_{n}\right\rangle$ be a finitely generated hyperbolic rational semigroup satisfying the strong open set condition. We assume that when $n$ is equal to one the degree of $f_{1}$ is at least two. Let $\delta$ be a number satisfying that $0<\delta \leq 2$ and assume that there is a $\delta$-conformal measure $\mu$ on $J(G)$. Then $\delta=\delta(G)$ and

$$
\operatorname{dim}_{H}(J(G))=\delta(G), \quad 0<H_{\delta(G)}(J(G))<\infty,
$$

where $\operatorname{dim}_{H}$ is the Hausdorff dimension and $H_{\alpha}$ is the $\alpha$-Hausdorff measure.

By Theorem 2.10, Theorem 3.2 and Theorem 3.4, we get the next result. 
COROLlaRY 3.5. Let $G=\left\langle f_{1}, f_{2}, \ldots, f_{n}\right\rangle$ be a finitely generated hyperbolic rational semigroup satisfying the strong open set condition. We assume that when $n$ is equal to one the degree of $f_{1}$ is at least two. Then

$$
0<\operatorname{dim}_{H}(J(G))<2 .
$$

And if we set $\alpha=\operatorname{dim}(J(G))$, then

$$
0<H_{\alpha}(J(G))<\infty .
$$

COROLlaRY 3.6. Let $G=\left\langle f_{1}, f_{2}, \ldots, f_{n}\right\rangle$ be a finitely generated hyperbolic rational semigroup. We assume that when $n$ is equal to one the degree of $f_{1}$ is at least two and the sets $\left\{f_{j}^{-1}(J(G))\right\}_{j=1, \ldots, n}$ are mutually disjoint. Then

$$
0<\operatorname{dim}_{H}(J(G))<2 .
$$

And if we set $\alpha=\operatorname{dim}(J(G))$, then

$$
0<H_{\alpha}(J(G))<\infty .
$$

Proof of Corollary 3.6. By Lemma 1.5.1 and Theorem 2.6, we can assume that

$$
\inf _{J} \inf _{z \in f_{j}^{-1}(J(G))}\left\|f_{J}^{\prime}(z)\right\|>1
$$

where we denote by $\|\cdot\|$ the norm of the derivative with respect to the spherical metric. Then it is easy to see that $G$ satisfies the strong open set condition. Now the statement follows from Corollary 3.5.

Proof of Theorem 3.4. To prove our theorem it is sufficient to show that if for a number $\delta$ satisfying $0<\delta \leq 2$ there is a $\delta$-conformal measure $\mu$ on $J(G)$, then $0<H_{\delta}(J(G))<\infty$. We set

$$
\lambda=\inf _{J} \inf _{z \in f_{j}^{-1}(J(G))}\left\|f_{J}^{\prime}(z)\right\| .
$$

By Lemma 1.5.1 and Theorem 2.6 we can assume that $\lambda>1$ by replacing $G$ by a subsemigroup $I_{m}$ of $G$. As $G$ is hyperbolic, there is a number $r>0$ such that for each $\zeta \in J(G)$ and for each $g \in G$ we can take well defined branches of $g^{-1}$ on $D(\zeta, r)$ where $D(\zeta, r)$ is the $r$ disc about $\zeta$. Also we can assume that for each $j$ and for each $\zeta \in J(G)$ the map $f_{j}$ is injective on $D(\zeta, r)$. We set

$$
\mathscr{S}_{\zeta}=\left\{S \mid \text { a branch of } g^{-1} \text { on } D(\zeta, r), g \in G\right\} \text {. }
$$

By the Koebe theorem, there is a positive number $c_{0}$ such that for each $\zeta \in J(G)$ and for each $S \in \mathscr{S}_{\zeta}$

$$
\sup \left\{\left\|S^{\prime}(z)\right\| \mid z \in D\left(\zeta, \frac{r}{2}\right)\right\} \leq c_{0} \cdot \inf \left\{\left\|S^{\prime}(z)\right\| \mid z \in D\left(\zeta, \frac{r}{2}\right)\right\}
$$

We fix a point $z_{0} \in J(G)$. For each positive integer $n$ there is a unique element 
$g_{n} \in G$ of word length $n$ such that $g_{n}\left(z_{0}\right) \in J(G)$ because of Lemma 1.4.2 and the strong open set condition. We take a branch $S_{n}$ of $g_{n}^{-1}$ such that $S_{n}\left(g_{n}\left(z_{0}\right)\right)=$ $z_{0}$. By the Koebe theorem there are a positive constant $\alpha, \beta$ such that if we set

$$
r_{n}=\frac{r \cdot\left\|\left(S_{n}\right)^{\prime}\left(z_{n}\right)\right\|}{\alpha},
$$

for each $n$ where $z_{n}=g_{n}\left(z_{0}\right)$, then

$$
\begin{aligned}
& D\left(z_{0}, r_{n}\right) \subset S_{n}\left(D\left(z_{n}, r\right)\right), \\
& D\left(z_{0}, r_{n}\right) \supset S_{n}\left(D\left(z_{n}, \frac{r}{\beta}\right)\right) .
\end{aligned}
$$

Also we have $r_{n} \rightarrow 0$ as $n \rightarrow \infty$. Since the support of $\mu$ is equal to $J(G)$, for each small number $a>0$ there is a number $M(a)>0$ such that for each $\zeta \in J(G)$

$$
\mu(D(\zeta, a))>M(a) .
$$

From above and since $\mu$ is $\delta$ conformal we get

$$
\begin{aligned}
\mu\left(D\left(z_{0}, r_{n}\right)\right) & \geq c_{0}^{-\delta}\left\|\left(S_{n}\right)^{\prime}\left(z_{n}\right)\right\|^{\delta} \mu\left(D\left(z_{n}, \frac{r}{\beta}\right)\right) \\
\geq & \left(\frac{r c_{0}}{\alpha}\right)^{-\delta} r_{n}^{\delta} M\left(\frac{r}{\beta}\right), \\
\mu\left(D\left(z_{0}, r_{n}\right)\right) & \leq c_{0}^{\delta}\left|\left(S_{n}\right)^{\prime}\left(z_{n}\right)\right|^{\delta} \mu\left(D\left(z_{n}, r\right)\right) \\
& \leq c_{0}^{\delta}\left(\frac{r}{\alpha}\right)^{-\delta} r_{n}^{\delta} .
\end{aligned}
$$

So there is a number $c_{1}>1$ such that for each $n$

$$
c_{1}^{-1} \leq \frac{\mu\left(D\left(z_{0}, r_{n}\right)\right)}{r_{n}^{\delta}} \leq c_{1} .
$$

We can take $c_{1}$ independent of $z_{0} \in J(G)$. We set

$$
c_{2}=\frac{\max _{J} \max _{z \in f_{j}^{-1}(J(G))}\left\|f_{J}^{\prime}(z)\right\|}{\lambda} .
$$

There is a number $n$ such that

$$
c_{2}^{-1} r_{n} \leq r^{\prime} \leq c_{2} r_{n},
$$

for all $r^{\prime}$ with $r^{\prime}<r_{1}$. Then for each $r^{\prime}$ such that $r^{\prime}<r_{1}$

$$
\begin{gathered}
\mu\left(D\left(z_{0}, r_{n}\right)\right) \geq c_{1}^{-1} r_{n}^{\delta} \geq\left(c_{1} c_{2}^{\delta}\right)^{-1}\left(r^{\prime}\right)^{\delta} \\
\mu\left(D\left(z_{0}, r_{n}\right)\right) \leq c_{1} r_{n}^{\delta} \leq c_{1} c_{2}^{\delta}\left(r^{\prime}\right)^{\delta}
\end{gathered}
$$


So if we set $c=c_{1} c_{2}^{\delta}$, for any small $r^{\prime}$

$$
c^{-1}\left(r^{\prime}\right)^{\delta} \leq \mu\left(D\left(z_{0}, r^{\prime}\right)\right) \leq c\left(r^{\prime}\right)^{\delta} .
$$

Now the statement of our theorem follows immediately.

In [DU], M. Denker and M. Urbański gave a conjecture that for any rational map $f, \operatorname{dim}_{H}(J(\langle f\rangle))=\delta(\langle f\rangle)$. Similary we give the following conjecture.

CONJECTURE 3.7. Let $G=\left\langle f_{1}, f_{2}, \ldots, f_{n}\right\rangle$ be a finitely generated rational semigroup satisfying the strong open set condition. We assume that when $n$ is equal to one the degree of $f_{1}$ is at least two. Then

$$
\operatorname{dim}_{H}(J(G))=\delta(G) .
$$

\section{4. $\delta$-subconformal measure}

DEFINITION 4.1. Let $G$ be a rational semigroup and $\delta$ be a non-negative number. We say that a probability measure $\mu$ on $\bar{C}$ is $\delta$-subconformal if for each $g \in G$ and for each measurable set $A$,

$$
\mu(g(A)) \leq \int_{A}\left\|g^{\prime}(z)\right\|^{\delta} d \mu .
$$

For each $x \in \bar{C}$ and each real number $s$ we set

$$
S(s, x)=\sum_{g \in G} \sum_{g(y)=x}\left\|g^{\prime}(y)\right\|^{-s}
$$

counting multiplicities and

$$
S(x)=\inf \{s \mid S(s, x)<\infty\}
$$

If there is not $s$ such that $S(s, x)<\infty$, then we set $S(x)=\infty$. Also we set

$$
s_{0}(G)=\inf \{S(x)\}, \quad s(G)=\inf \{\delta \mid \exists \mu: \delta \text {-subconformal measure }\} .
$$

By using the same method of the proof of Theorem 3.2, we can show the following result.

THEOREM 4.2. Let $G$ be a rational semigroup which has at most countably many elements. If there exists a point $x \in \bar{C}$ such that $S(x)<\infty$ then there is a $S(x)$-subconformal measure.

Proposition 4.3. Let $G$ be a rational semigroup and $\tau$ a $\delta$-subconformal measure for $G$ where $\delta$ is a real number. Assume that $\sharp J(G) \geq 3$ and for each $x \in E(G)$ there exists an element $g \in G$ such that $g(x)=x$ and $\left|g^{\prime}(x)\right|<1$. Then the support of $\tau$ contains $J(G)$. 
Proof. Assume that there are a point $\zeta \in J(G)$ and a positive number $a$ such that $\tau(D(\zeta, a))=0$. By Lemma 1.5, for each neighborhood $U$ of $E(G)$ there exists an element $h \in G$ such that $h(D(\zeta, a)) \supset \bar{C} \backslash U$. Since $\tau$ is a subconformal measure, it follows that $\tau(\bar{C} \backslash E(G))=0$. From the assumption of our theorem, we have $\tau(E(G))=0$ and so $\tau(\bar{C})=0$ but this is a contradiction.

THEOREM 4.4. Let $G=\left\langle f_{1}, f_{2}, \ldots, f_{n}\right\rangle$ be a finitely generated rational semigroup. Assume that $G$ is expanding. Then $s_{0}(G)<\infty$ and

$$
\operatorname{dim}_{H}(J(G)) \leq s(G) \leq s_{0}(G) .
$$

Proof. Because $G$ is expanding, it is easy to see that $s_{0}(G)<\infty$. We will show the statement in the same way as the proof of Theorem 3.4. Since we have only to consider the case that $\sharp J(G) \geq 3$, we assume that. We set $\delta=s(G)$. Let $\mu$ be a $\delta$-subconformal measure.

First we will show that the support of $\mu$ contains $J(G)$. To show that, from Proposition 4.3, we have only to show that for each $x \in E(G)$ there exists an element $g \in G$ such that $g(x)=x$ and $\left|g^{\prime}(x)\right|<1$. If there exists an element of $G$ with the degree at least two, then it is easy to show that. Now consider the case such that each element of $G$ is of degree one. Since $G$ is expanding, the order of each element of $G$ is infinite. From Lemma $1.5, \sharp E(G) \leq 2$. Let $x$ be any point of $E(G)$. Let $g_{1}, \ldots, g_{n^{2}}$ be all elements of $G$ each of which is in the form $f_{i_{1}} \circ f_{i_{2}}$. Then for each $j=1, \ldots, n^{2}, g_{j}(x)=x$. Assume that for each $j=$ $1, \ldots, n^{2},\left|g_{j}^{\prime}(x)\right| \geq 1$. Since $G$ is expanding, for each $j=1, \ldots, n^{2},\left|g_{j}^{\prime}(x)\right|>1$. With this fact, from Lemma 1.5.5, $x$ is an isolated point of $J(G)$. On the other hand, from Lemma 1.5.2, $J(G)$ is a perfect set and this is a contradiction. So there is a number $j$ such that $\left|g_{j}^{\prime}(x)\right|<1$. Hence the support of $\mu$ contains $J(G)$.

Next let $z_{0} \in J(G)$ be any point. Because of the backward self-similarity of $J(G)$, we have for each positive integer $m$, there is an element $g_{m} \in G$ which is a product of $m$ generators such that $g_{m}\left(z_{0}\right) \in J(G)$. As in the proof of Theorem 3.4, with the fact that the support of $\mu$ contains $J(G)$, we can show that there is a sequence $\left(r_{m}\right)$ of positive numbers converging to zero as $m \rightarrow \infty$ such that for each $m$,

$$
c \leq \frac{\mu\left(D\left(z_{0}, r_{m}\right)\right)}{r_{m}^{\delta}}
$$

where $c$ is a positive constant independent of $z_{0} \in J(G)$ and $m$. Hence the statement of our theorem follows.

THEOREM 4.5. Let $G=\left\langle f_{1}, f_{2}, \ldots, f_{n}\right\rangle$ be a finitely generated rational semigroup which is expanding. Let $\lambda$ be the number in the assumption of Theorem 2.8. Then

$$
\operatorname{dim}_{H}(J(G)) \leq \frac{\log \left(\sum_{j} \operatorname{deg}\left(f_{j}\right)\right)}{\log \lambda}
$$


Proof. By replacing $G$ by a subsemigroup $I_{m}$ of $G$, we can assume that for each $j$ and $z \in f_{j}^{-1}(J(G))$

$$
\left\|f_{j}^{\prime}(z)\right\| \geq \lambda,
$$

where $\|\cdot\|$ denotes the norm of the derivative with respect to the spherical metric. We take a point $x \in J(G)$. Now for each $m$ the set

$$
\bigcup_{g \in G: \text { word length } m} g^{-1}\{x\}
$$

has at most $\left(\sum_{j}\left(\operatorname{deg}\left(f_{j}\right)\right)\right)^{m}$ points. Also for each $z \in J(G)$ and for each $m$, if $g \in G$ is word length $m$ and $g(z) \in J(G)$, then

$$
\left\|g^{\prime}(z)\right\| \geq \lambda^{m} \text {. }
$$

So for each number $s$ such that $s>\log \left(\sum_{j} \operatorname{deg}\left(f_{j}\right)\right) / \log \lambda$ we have

$$
\begin{aligned}
& \sum_{g \in G} \sum_{g(y)=x}\left\|g^{\prime}(y)\right\|^{-s} \leq \sum_{m=0}^{\infty}\left(\sum_{J} \operatorname{deg}\left(f_{j}\right)\right)^{m} \lambda^{-m s} \\
& \quad=\sum_{m=0}^{\infty} \exp \left\{m \log \left(\sum_{J} \operatorname{deg}\left(f_{j}\right)\right)\left(1-s \frac{\log \lambda}{\log \left(\sum_{j} \operatorname{deg}\left(f_{j}\right)\right)}\right)\right\}<\infty .
\end{aligned}
$$

From the way of construction of $\delta$-subconformal measure and Theorem 4.4, the statement of Theorem 4.5 follows.

Remark 5. In the sequel [S5], we will show (6) by using a method of the thermodynamic formalism.

EXAMPLE 4.6. Let $n$ be a positive integer such that $n \geq 4$. We set $G=$ $\left\langle z^{n}, n(z-4)+4\right\rangle$. Then $G$ is a finitely generated hyperbolic rational semigroup satisfying the strong open set condition. For, let $f(z)=z^{n}, g(z)=n(z-4)+4$ and $U=\{|z|<5\}$. Then the closures of $f^{-1}(U)$ and $g^{-1}(U)$ are included in $U$ and mutually disjoint. Hence $J(G) \subset U$ and $G$ satisfies the strong open set condition. Since $|g(0)|>5, G$ is hyperbolic. By Theorem 4.5, we get

$$
1 \leq \operatorname{dim}_{H} J(G) \leq \frac{\log (n+1)}{\log (n)} .
$$

\section{REFERENCES}

[DU] M. DenKER AND M. URBaŃsKI, The dichotomy of Hausdorff measure and equilibrium states for parabolic rational maps. Ergodic Theory and Related Topics III, Proceedings Güstow 1990, eds. U. Krengel, K. Richter and V. Warstat, Lecture Notes in Math., 1514, Springer, Berlin, 1992, 90-113.

[HM1] A. HinkKanen AND G. J. MARTIN, The dynamics of semigroups of rational functions I, Proc. London Math. Soc. (3), 73 (1996), 358-384. 
[HM2] A. HinkKanen AND G. J. Martin, Julia sets of rational semigroups, Math. Z., 222 (1996), 161-169.

[M] C. McMullen, Complex Dynamics and Renormalization, Ann. of Math. Stud., 135, Princeton University Press, Princeton, New Jersey, 1994.

[MTU] S. Morosawa, M. Taniguchi and T. Ueda, A Primer on Complex Dynamics, (Japanese version; Baihuukan, 1995) English version, in preparation.

[P] S. J. Patterson, The limit set of a Fuchsian group, Acta Math., 136 (1976), 241-273.

[ZR] W. ZHOU AND F. REN, The Julia sets of the random iteration of rational functions, Chınese Bulletin, 37 (1992), 969-971.

[GR] Z. GoNG AND F. REN, A random dynamical system formed by infinitely many functions, J. Fudan Univ. Nat. Sci., 35 (1996), 387-392.

[Sul] D. Sullivan, Conformal dynamical system, Geometric Dynamics, Lecture Notes in Math., 1007, Springer, 1983, 725-752.

[S1] H. Sum, On limit functions and continuity of Julia sets of rational semigroups, Complex Dynamics and Related Problems, Sūrikaisekikenkyūsho Kōkyūroku, 959 (1996), 59-72 (in Japanese).

[S2] H. Sumi, On dynamics of hyperbolic rational semigroups and Hausdorff dimension of Julia sets, Complex Dynamics and Related Problems, Sūrikaisekikenkyūsho Kōkyūroku, 988 (1997), 98-113.

[S3] H. Sum, On dynamics of hyperbolic rational semigroups, to appear in J. Math. Kyoto Univ.

[S4] H. SumI, Invariant measures and Hausdorff dimension of Julia sets of rational semigroups, preprint.

[S5] H. Sum, Conformal measures and self-similar measures of rational semigroups, in preparation.

[S6] H. SumI, Topological pressure and conformal measures in semigroup dynamics, Sci. Bull. Josai Univ., Special Issue no. 4, 1998, 29-46.

[S7] H. SumI, Dynamics of sub-hyperbolic and semi-hyperbolic rational semigroups and conformal measures of rational semigroups, Complex Dynamics and Related Problems, to appear in Sūrikaisekikenkyūsho Kōkyūroku.

[S8] H. Sum, Dynamics of sub-hyperbolic and semi-hyperbolic rational semigroups, in preparation.

\author{
Graduate School of Human \\ AND ENVIRONMENTAL STUdies \\ KYOTO UNIVERSITY, KYOTO 606-8501 \\ JAPAN \\ e-mail: sumi@math.h.kyoto-u.ac.jp
}

